\title{
AIP
}

\section{Gas-phase structures of neutral silicon clusters}

Marko Haertelt, Jonathan T. Lyon, Pieterjan Claes, Jorg de Haeck, Peter Lievens et al.

Citation: J. Chem. Phys. 136, 064301 (2012); doi: 10.1063/1.3682323

View online: http://dx.doi.org/10.1063/1.3682323

View Table of Contents: http://jcp.aip.org/resource/1/JCPSA6/v136/i6

Published by the American Institute of Physics.

\section{Related Articles}

Mg impurity in helium droplets

JCP: BioChem. Phys. 6, 02B602 (2012)

Mg impurity in helium droplets

J. Chem. Phys. 136, 054301 (2012)

Mid-infrared spectroscopy of molecular ions in helium nanodroplets

J. Chem. Phys. 136, 044305 (2012)

Vibrational energy relaxation of benzene dimer and trimer in the $\mathrm{CH}$ stretching region studied by picosecond time-resolved IR-UV pump-probe spectroscopy

J. Chem. Phys. 136, 044304 (2012)

Influence of the cluster dimensionality on the binding behavior of $\mathrm{CO}$ and $\mathrm{O} 2$ on Au13

J. Chem. Phys. 136, 024312 (2012)

\section{Additional information on J. Chem. Phys.}

Journal Homepage: http://jcp.aip.org/

Journal Information: http://jcp.aip.org/about/about_the_journal

Top downloads: http://jcp.aip.org/features/most_downloaded

Information for Authors: http://jcp.aip.org/authors

\section{ADVERTISEMENT}

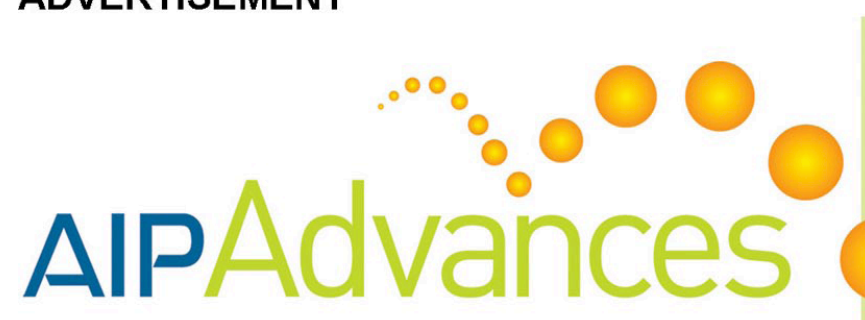

Submit Now
Explore AIP's new open-access journal

Article-level metrics now available

Join the conversation! Rate $\&$ comment on articles 


\title{
Gas-phase structures of neutral silicon clusters
}

\author{
Marko Haertelt, ${ }^{1}$ Jonathan T. Lyon, ${ }^{2, a)}$ Pieterjan Claes, ${ }^{3}$ Jorg de Haeck, ${ }^{3}$ Peter Lievens, $\left.{ }^{3, b}\right)$ \\ and André Fielicke ${ }^{1, c)}$ \\ ${ }^{1}$ Fritz-Haber-Institut der Max-Planck-Gesellschaft, Faradayweg 4-6, D-14195 Berlin, Germany \\ ${ }^{2}$ Department of Natural Sciences, Clayton State University, 2000 Clayton State Blvd., Morrow, \\ Georgia 30260, USA \\ ${ }^{3}$ Laboratory of Solid State Physics and Magnetism, KU Leuven, Celestijnenlaan 200 D, \\ $B$-3001 Leuven, Belgium
}

(Received 3 November 2011; accepted 17 January 2012; published online 9 February 2012)

\begin{abstract}
Vibrational spectra of neutral silicon clusters $\mathrm{Si}_{n}$, in the size range of $n=6-10$ and for $n=15$, have been measured in the gas phase by two fundamentally different IR spectroscopic methods. Silicon clusters composed of 8,9 , and 15 atoms have been studied by IR multiple photon dissociation spectroscopy of a cluster-xenon complex, while clusters containing 6, 7, 9, and 10 atoms have been studied by a tunable IR-UV two-color ionization scheme. Comparison of both methods is possible for the $\mathrm{Si}_{9}$ cluster. By using density functional theory, an identification of the experimentally observed neutral cluster structures is possible, and the effect of charge on the structure of neutrals and cations, which have been previously studied via IR multiple photon dissociation, can be investigated. Whereas the structures of small clusters are based on bipyramidal motifs, a trigonal prism as central unit is found in larger clusters. Bond weakening due to the loss of an electron leads to a major structural change between neutral and cationic $\mathrm{Si}_{8}$. () 2012 American Institute of Physics. [doi:10.1063/1.3682323]
\end{abstract}

\section{INTRODUCTION}

Silicon based semiconductor technology is the framework of our technological evolution. Further and further miniaturizing allowed developing faster and faster processors and Moore's famous prediction ${ }^{1}$ remained valid for several decades. But the increasing costs in producing processors already foreshadow its limit. ${ }^{2}$ Today's transistors have sizes not far from molecules and clusters, and size-dependent effects will start to play a role for future nano-electronic devices. The top-down approach used to assemble current electronics will, in parts, be replaced by the bottom-up approach as can already be seen in molecular nano-electronic devices currently developed. ${ }^{3}$ At this transition region between the bulk and the molecular level, fundamental understanding and, more importantly, predictability is required to achieve sufficient reliability and reproducibility for large scale-production of nanoelectronic devices. ${ }^{2,4}$

Quantum chemistry aims for this and can describe the physical and chemical properties from the bulk to the molecular level. Despite its enormous progress, which runs in parallel with increasing demands on calculation power, sometimes such "simple" properties like structures cannot be easily obtained. Even though the potential energy surface can be mapped accurately nowadays by various methods, e.g., simulated annealing, global optimization or basin-hopping, the major limitation, however, is the missing knowledge about the required level of theory properly describing the system.

\footnotetext{
a) Electronic mail: JonathanLyon@clayton.edu.

b)Electronic mail: peter.lievens@fys.kuleuven.be.

c) Electronic mail: fielicke@fhi-berlin.mpg.de.
}

Experiments can provide additional insights and work as a reference for the calculations.

Because of the technological relevance of silicon, many experimental studies have focused on nano-scale Si particles and clusters. In the gas phase, where isolated bare (but also doped) silicon clusters can be studied and therefore act as an ideal model systems, the electronic as well as geometric properties of silicon clusters have been investigated, e.g., by resonance enhanced multiple photon electron detachment, ${ }^{5}$ anion photoelectron spectroscopy ${ }^{6-10}$ photoionization efficiency spectroscopy, ${ }^{11,12}$ ion mobility, ${ }^{13}$ and various other methods.

While most experiments have been concentrated on charged species, theory can be performed on all charge states. ${ }^{14-22}$ For smaller anionic and cationic clusters, the combination of experiments with high level theory makes it possible to determine the structures of these clusters. ${ }^{23-25}$ For medium-sized clusters, changes in the structural motif were suggested. Recently, we used infrared multiple photon dissociation (IR-MPD) to obtain vibrational spectra of cationic silicon clusters with 6 to 21 atoms. ${ }^{26}$ For many sizes up to the $\mathrm{Si}_{18}{ }^{+}$cluster a structural assignment based on the observed experimental IR spectra was possible. For larger sizes, an identification of the structure is still missing. However, theoretical endeavors continue to make new structural assignments based on the IR-MPD spectra. ${ }^{27}$

For neutral Si clusters, experimental data is spare and is mainly limited to ionization efficiency ${ }^{12}$ or bracketing measurements. ${ }^{11}$ The information on the ionization energy (IE) of a system can be used to rule out some isomers predicted by theory, but it is often not sufficient to decide for a single isomer. ${ }^{24}$ Only for smaller sizes, IR and Raman matrix 
isolation spectroscopy allowed for structural assignment up to $\mathrm{Si}_{7} \cdot{ }^{28,29}$ Vibrational spectroscopy of clusters in the gas phase suffers from the low sample densities achievable in molecular beams. The absorption of IR photons can be detected, however, indirectly by fragmentation or ionization processes and employs the high sensitivity of mass spectrometric methods. In this study, both kinds of processes have been employed in order to obtain spectra for a broader size range of silicon clusters. For silicon clusters containing 6, 7, and 10 atoms, we have recently applied a tunable IR-UV two-color ionization (IR-UV2CI) technique to obtain vibrational spectra for these clusters and thereby confirmed theoretical predictions. ${ }^{30}$ Here, we revisit these results and extend them with the results for $\mathrm{Si}_{9}$. With a different method, the IR-MPD of Xe messenger complexes, IR spectra of $\mathrm{Si}_{8}, \mathrm{Si}_{9}$, and $\mathrm{Si}_{15}$ have been measured and are reported here for the first time. For all investigated cluster sizes, structural assignments are made by comparison with results from density functional theory calculations.

\section{EXPERIMENTAL AND THEORETICAL METHODS}

An essential step in the mass spectrometric detection of neutral Si clusters is their ionization. For the clusters discussed here containing 6-10, and 15 silicon atoms Fuke et al. reported bracketing threshold photoionization measurements and determined IEs in the range of 7.17-7.9 eV. This is in the vacuum ultraviolet range, where no simple (tunable) high fluence laser sources exist. In all of our studies reported here, we applied an $\mathrm{F}_{2}$ laser with photon energy of $7.87 \mathrm{eV}$. For several of the cluster sizes this is close to, or even below, their reported IE and therefore a single VUV photon does not (efficiently) ionize these clusters. The different ionization efficiencies become directly apparent in the intensity modulation of the mass spectrum (Fig. 1(a)).

For both applied methods, IR-MPD and IR-UV2CI, bare silicon clusters are made by laser ablation from a continuously moving silicon plate by a focused Nd:YAG laser. ${ }^{31,32}$ The produced plasma is quenched in a short helium gas pulse

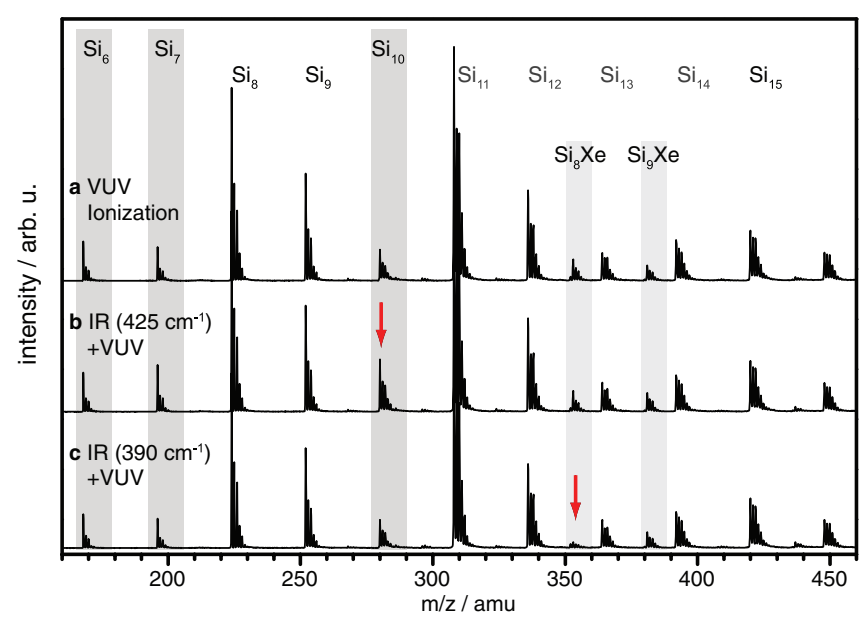

FIG. 1. Mass spectra of neutral Si clusters obtained under different ionization conditions. The upper panel shows a spectrum measured with the $\mathrm{F}_{2}$ ionization laser only, while the lower panels show spectra for a distribution irradiated with different frequencies of FELIX. mixed with $2 \%$ of isotopically enriched ${ }^{129} \mathrm{Xe}$. In a thermalization channel attached to the source the clusters are cooled to $100 \mathrm{~K}$. This quenches electronically and vibrationally excited states and is required for the formation of a messenger complex in the IR-MPD experiments. The molecular beam is first skimmed and then shaped with a flat aperture with a 1 $\mathrm{mm}$ opening. Ions are removed from the beam by a voltage applied to the aperture. Within the extraction region of a reflectron time-of-flight mass spectrometer, an unfocussed $\mathrm{F}_{2}$ laser is used for photoionization of the neutral cluster distribution. Species with an IE close to or above the photon energy of the ionization laser, e.g., $\mathrm{Si}_{6,7,9,10}$, have a lower intensity in the time-of-flight spectra. Only a residual signal from the hot fraction can be ionized and therefore detected. An IR spectrum of such species can be obtained by overlapping the molecular beam with the counter propagating IR beam from the Free Electron Laser for Infrared eXperiments (FELIX), which delivers $\sim 5 \mu$ s long pulses with energies of $20-40 \mathrm{~mJ}$. The IR beam is loosely focused $\sim 30 \mathrm{~mm}$ behind the aperture. Upon resonance with a vibrational mode, several photons can be absorbed leading to a raise in internal energy. In contrast to the previously cold cluster, these "hot" clusters can be more efficiently ionized (see $\mathrm{Si}_{10}$ in Fig. 1(b)). By monitoring the relative increase in intensity as a function of IR wavelength, an IR-UV2CI spectrum can be measured. The technique has been described previously ${ }^{30}$ and in more detail for $\mathrm{MgO}$ clusters. ${ }^{33}$

Clusters with an IE below $\sim 7.9 \mathrm{eV}$ can be ionized and therefore detected with mass spectrometry. For this group of clusters, e.g., $\mathrm{Si}_{8,9,15}$, the dissociation technique can be used, provided that the messenger complex, $\mathrm{Si}_{n}{ }^{129} \mathrm{Xe}$, can be formed (attachment of the Xe atom is expected to shift the IE to slightly lower values, see, e.g., Ref. 34). Again, in case of a resonance, several IR photons are absorbed, this time leading to evaporation of the xenon (see $\mathrm{Si}_{8} \mathrm{Xe}$ in Fig. 1(c)). This loss can be observed by taking mass spectra alternating with and without FELIX. By scanning the IR wavelength and monitoring the relative intensity of the rare gas complex, a depletion spectrum is measured, which can be transformed into an absorption spectrum. The experimental setup and methods are similar as those used for cationic silicon clusters. ${ }^{26}$

To assign the experimentally observed spectra to certain cluster structures, comparison with calculated IR spectra is required. Given that (neutral) silicon clusters have already been studied by many groups, often even with global optimization methods, a fairly well set of local minimum structures is available. ${ }^{18-24}$ Care has to be taken with regards to which method is used for the prediction of the IR spectrum of different cluster structures. Recently, we have shown that density functional theory (DFT) and second-order MøllerPlesset (MP2) methods cannot only predict different global minima, but more importantly, can give different IR spectra for the same relaxed cluster structure as has been exemplified for $\mathrm{Si}_{11}+{ }^{26}$ However, for most small and mediumsized silicon cluster cations and neutrals, DFT with the BP-86 functional ${ }^{35,36}$ and the SVP basis set $^{37}$ using the resolution of identity $(\mathrm{RI})$ approximation within the GAUSSIAN03 program suite ${ }^{38}$ turned out to be sufficient and therefore was used in this work. No symmetry constraints 
were applied for the systems studied here. Relative energies of different isomers determined by DFT are given in $\mathrm{kJ} / \mathrm{mol}$ and include zero point vibrational energy corrections. The messenger atom has not been explicitly considered, as the interaction between the neutral $\mathrm{Si}$ clusters and the Xe atoms is expected to be significantly weaker than for the cationic $\mathrm{Si}$ clusters that have calculated Xe binding energies lower than $0.1 \mathrm{eV}^{26}$ The electronic state of all clusters corresponds to the lowest possible spin multiplicity. The calculated IR frequencies have been rescaled with a multiplication factor of 1.03 , as before, ${ }^{26,30}$ and have been folded with a Gaussian function with a full width at half maximum of $8 \mathrm{~cm}^{-1}$. The experimental spectra have been smoothed with a 5-point running average. All spectra are corrected by the photon flux, which often gives better agreement with theory, ${ }^{33}$ compared to the previously applied correction by the pulse energy only. ${ }^{39}$

\section{RESULTS AND DISCUSSION}

The results for $\mathrm{Si}_{6,7,10}$ have been reported before ${ }^{30}$ and are summarized here for completeness. For these three systems the IEs are close to the UV photon energy and the IR spectra are determined via IR-UV2CI (see Fig. 2). For these and all other sizes considered in the following, the identified global minimum structures provide the best fit to the experiment. Only for $\mathrm{Si}_{6}$ does DFT and MP2 methods predict dif-

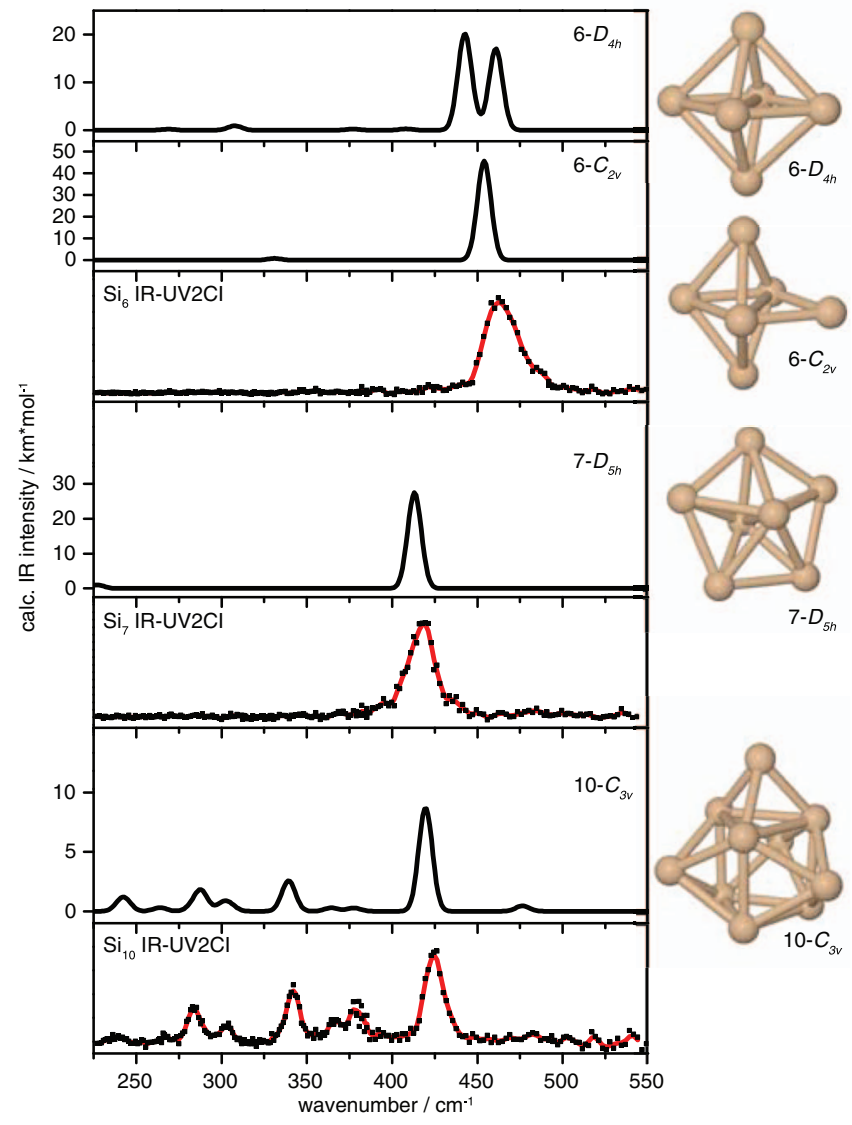

FIG. 2. IR-UV2CI spectra of $\mathrm{Si}_{6}, \mathrm{Si}_{7}$, and $\mathrm{Si}_{10}$ clusters compared to infrared spectra of the lowest energy structures from DFT (and MP2 calculation for the $\mathrm{Si}_{6}$ ). In the experimental spectra reported here, the dots correspond to original data points folded with a 5-point binomial weighted average as a solid red line. The y-axis for the experimental spectrum has arbitrary units.

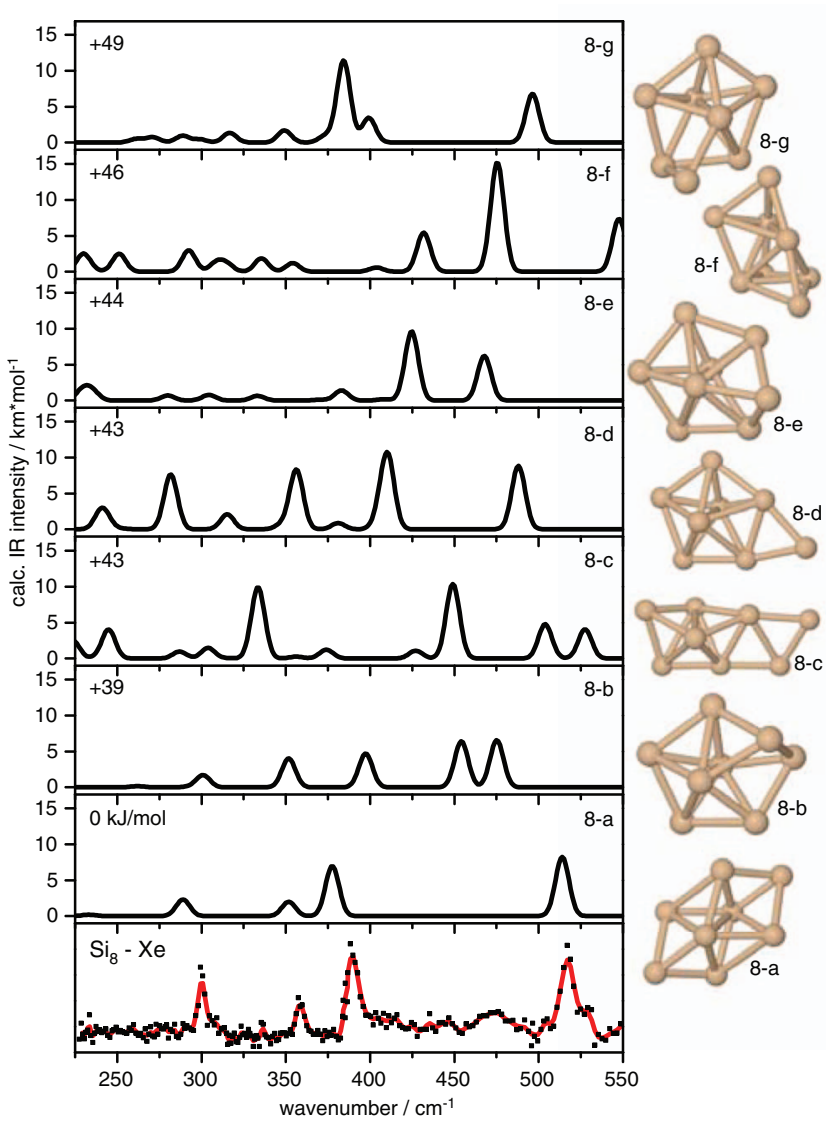

FIG. 3. IR-MPD spectrum of xenon-tagged $\mathrm{Si}_{8}$ cluster compared to infrared spectra of low lying isomers predicted by DFT. Relative energies are given in $\mathrm{kJ} / \mathrm{mol}$.

ferent ground state symmetries of the lowest energy structure. A more detailed description can be found in Ref. 30. Briefly, a $D_{4 h}$ tetragonal bipyramid structure as the putative global minimum has been found by the MP2 method, while using DFT, this structure gets perturbed to $C_{2 v}$ symmetry. Both structures have very similar linear absorption spectra and agree well with the experiment, see Fig. 2. This makes an unambiguous assignment to a single structure difficult. For $\mathrm{Si}_{7}$ the calculated linear absorption spectrum of a pentagonal bipyramid agrees best with the experimental spectrum. For both sizes the structure gets perturbed, when going to the cationic system, but still resembles the same structural motif. A comparison between the assigned cationic and neutral cluster structures can be found in Fig. 7. The discussion for $\mathrm{Si}_{10}$ is presented in detail later.

For $\mathrm{Si}_{8}$ Fig. 3 shows the measured IR-MPD spectrum of its Xe complex and calculated linear absorption spectra for several low lying isomers. The putative global minimum structure, 8-a, is a bicapped octahedron structure, $39 \mathrm{~kJ} / \mathrm{mol}$ below the next isomer. All four experimentally observed bands at $300,359,390$, and $517 \mathrm{~cm}^{-1}$ are reproduced by the calculated spectrum, which predicts stronger absorption at $289,352,378$, and $514 \mathrm{~cm}^{-1}$. Most of the other isomers predicted emerge from differently capping a pentagonal bipyramid, and none of them explains the observed experimental IR spectrum. For the cationic structure, an edgecapped pentagonal bipyramid structure was identified, and 

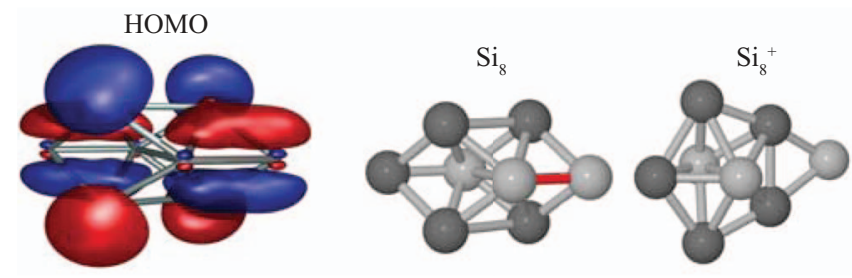

FIG. 4. HOMO of the neutral $\mathrm{Si}_{8}$ and a detailed comparison of the structures of cationic and neutral $\mathrm{Si}_{8}$. In the neutral cluster, the HOMO has bonding character along the bond marked in red. Upon ionization, this level gets partially depopulated and the structure becomes unstable. The atoms forming the pentagonal ring are colored in dark grey.

this structure was also considered for the neutral system. This isomer 8 - $\mathrm{d}$ is much higher in energy $(+43 \mathrm{~kJ} / \mathrm{mol}) . \mathrm{Si}_{8}$ is a unique cluster size where a major difference between cationic and neutral cluster structure is observed. Such a behavior was already predicted, e.g., by Ref. 24, although a slightly different cationic structure was suggested as the putative minimum. A comparison of the cationic and neutral structures reveals that both can interconvert with only little atomic rearrangement, see Fig. 4. This structural change may be rationalized by the character of the highest occupied molecular orbital (HOMO) of $\mathrm{Si}_{8}$ which has bonding character along the $\mathrm{Si}-\mathrm{Si}$ bond that needs to break up to form the cationic structure; ionization facilitates the destabilization of this $\mathrm{Si}-\mathrm{Si}$ bond in the neutral (marked in red in Fig. 4).

The $\mathrm{Si}_{9}$ cluster is a system for which IR spectra could be obtained by both methods, IR-MPD and IR-UV2CI. The IR induced enhancement of the ionization efficiency is, however, less pronounced than for the clusters discussed before, which can be related to its lower IE. This is estimated to be in the range of 7.46-7.87 eV (for $\mathrm{Si}_{8}$ as well as for $\mathrm{Si}_{9}$ ) while for $\mathrm{Si}_{6,7,10}$ a value of about $7.9 \mathrm{eV}$ has been reported. ${ }^{11}$ These values are in accord with the mass spectra taken with the $F_{2}$ laser ionization, see Fig. 1, that shows for the latter sizes a lower intensity. However, the intensity of $\mathrm{Si}_{8}$ is almost a factor of two larger than for $\mathrm{Si}_{9}$, which is an indication for significant differences in the IEs of these two systems. Most probably, the IE of $\mathrm{Si}_{9}$ is closer to the upper limit of the range given by Fuke et al. More recent measurements of the ionization efficiency curves using synchrotron radiation showed some differences to the earlier experiments, in most cases the determined IEs are lower. ${ }^{12}$ In particular for $\mathrm{Si}_{2,3}$ quite large deviations, up to $0.5 \mathrm{eV}$, have been observed. However, these experiments strongly rely on the preparation of cold clusters and there seem to be differences in the thermalization of the clusters in the two experiments. Unfortunately, these results are limited to $n \leq 7$. Because of the lack of sufficiently precise measurements of the IEs for the larger systems, we have to rely on calculated vertical ionization energies (VIEs) to obtain information on the trend of the IE. The VIEs for $\operatorname{Si}_{6}\left(D_{4 h}\right.$ structure) and $\mathrm{Si}_{7}$ are 8.20 and $8.21 \mathrm{eV}$, respectively. They compare reasonably well with the experimentally determined adiabatic values of $7.8 \pm 0.1 \mathrm{eV}$, taken from Ref. 12. The calculated VIEs for $\mathrm{Si}_{9}$ and $\mathrm{Si}_{10}$ are $8.14 \mathrm{eV}$ and $8.11 \mathrm{eV}$, respectively, which is very similar, though the experiment suggests a slightly lower value for $\mathrm{Si}_{9}$ compared to $\mathrm{Si}_{10}$, while the IE for $\mathrm{Si}_{8}$ is significantly lower $(7.50 \mathrm{eV})$. Overall, this agrees well

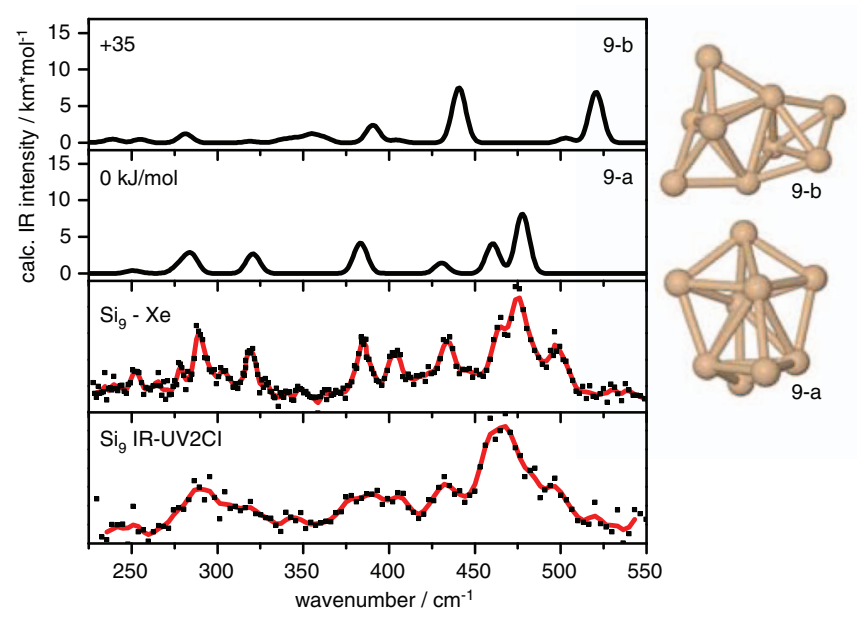

FIG. 5. IR-MPD and IR-UV2CI spectrum of Sig. Two low lying isomers and their predicted IR spectra are shown for comparison.

with the intensity pattern in the experimental mass spectrum obtained using an $\mathrm{F}_{2}$ laser for ionization.

For the $\mathrm{Si}_{9}$ cluster, the IE seems to be just low enough compared to the photon energy for efficient ionization of the xenon complex, albeit still in the rising wing of the photoionization efficiency curve, below saturation. This allows for the application of IR-MPD spectroscopy on the rare gas complex while also an IR-UV2CI spectrum can be observed. The latter spectrum is measured without any Xe gas added, and therefore it can be excluded that the enhancements stem from the fragmentation of the complex. The spectra measured by the two different methods are shown in Fig. 5. Both spectra look quite similar, however, the IR-MPD spectrum is better resolved and more features are observed. This is probably due to the lower internal energy required to dissociate the xenon complex vs. the bare cluster.

The calculated isomer showing the best agreement with the experiment, a bicapped pentagonal bipyramid, has the same structure as in the case of the cation. Most features are explained by this structure, except for bands at 403 and $497 \mathrm{~cm}^{-1}$. These features can be found in both experimental spectra, and can therefore not be attributed to a messenger effect. The feature at $403 \mathrm{~cm}^{-1}$ may come from a calculated mode at $403 \mathrm{~cm}^{-1}$, which has only a very weak predicted IR intensity of $0.03 \mathrm{~km} / \mathrm{mol}$, but may be enhanced in the experiment. Around $500 \mathrm{~cm}^{-1}$ no vibrational fundamental is predicted and also the isomers higher in energy do not have intense features at these frequencies (see Fig. 5 and supplementary information for additional isomers ${ }^{40}$ ). It may well be that another isomer is additionally present that has not been identified in the calculations. Nevertheless, it is noted that the depletion observed at $497 \mathrm{~cm}^{-1}$ amounts to $50 \%$ and for the most intense peak at $475 \mathrm{~cm}^{-1}$ to $80 \%$ of the total intensity of $\mathrm{Si}_{9} \mathrm{Xe}$. This may suggest that an additional isomer contributes significantly to the band at $475 \mathrm{~cm}^{-1}$.

For $\mathrm{Si}_{10}$ the trigonal prism, an important structural motif for larger Si clusters, becomes the structural base. Comparison between the IR-UV2CI spectrum and the calculated linear absorption spectrum of the fourfold capped trigonal prism structure of $C_{3 v}$ symmetry shows good agreement (see Fig. 2). 

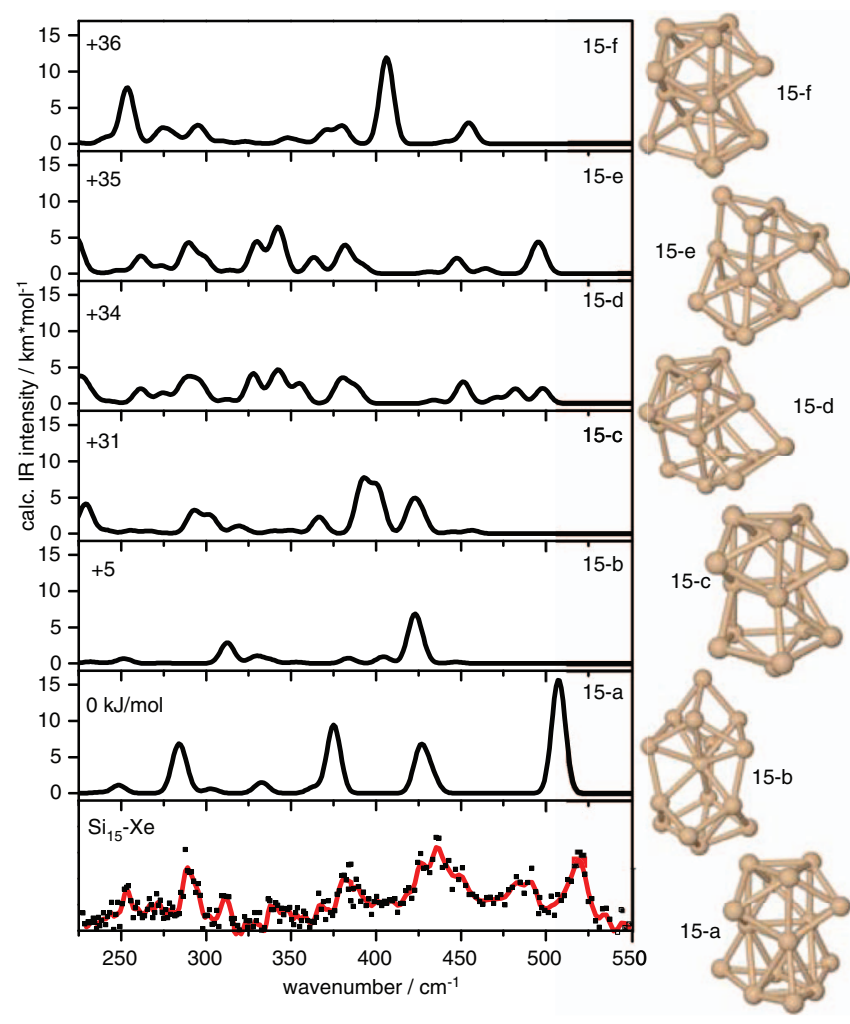

FIG. 6. IR-MPD spectrum of xenon-tagged $\mathrm{Si}_{15}$ clusters compared with linear absorption spectra for several predicted minimum energy structures.

Also for the cationic silicon clusters with 10 atoms this structure was observed. First indications for a new structural motif emerging at $\mathrm{Si}_{10}$ were found already in ion mobility experiments, where a sudden jump in the mobilities was observed. ${ }^{24}$ Later IR-MPD experiments by our group ${ }^{26}$ confirmed these findings and proved the trigonal prism as structural subunit for cationic $\mathrm{Si}_{11,13,15}$, and $\mathrm{Si}_{18}$.

Unfortunately, even though $\mathrm{Si}_{11}$ to $\mathrm{Si}_{14}$ were observed in the mass spectra and their IEs are well below the photon energy of the $\mathrm{F}_{2}$ laser, the intensities for the xenon complexes were too low to obtain IR spectra for these clusters. Only for the $\mathrm{Si}_{15}$ cluster was the complex intensity sufficient, and the experimental IR-MPD spectrum can be seen in Fig. 6. The lowest energy isomer found in the calculations is the same as for the cationic system, see Fig. 7. In both cases, a tricapped trigonal prism with a six-atom triangle attached, isomer $15-\mathrm{a}$, is predicted as the global minimum structure. All major features of the IR-MPD spectrum are reproduced. Also, the broader band in the range $425-450 \mathrm{~cm}^{-1}$, which seems to contain several features, may be explained by this isomer. Although the calculated spectrum appears as a single band, it is composed of three close-lying modes at 425.9, 426.2, and $432.6 \mathrm{~cm}^{-1}$. Another explanation for this broad band might be the additional presence of the second lowest energy structure $15-\mathrm{b}$, which is $5 \mathrm{~kJ} / \mathrm{mol}$ above the putative global minimum. For this structure, the calculation predicts an intense absorption at $422 \mathrm{~cm}^{-1}$, and two weaker bands at 425 and $426 \mathrm{~cm}^{-1}$. Also other bands may have a counterpart in the experimental spectrum. This may be the first evidence for the prediction that for neutral silicon clusters with more than 12 atoms several isomers are present at elevated temperatures. ${ }^{41}$ Only a

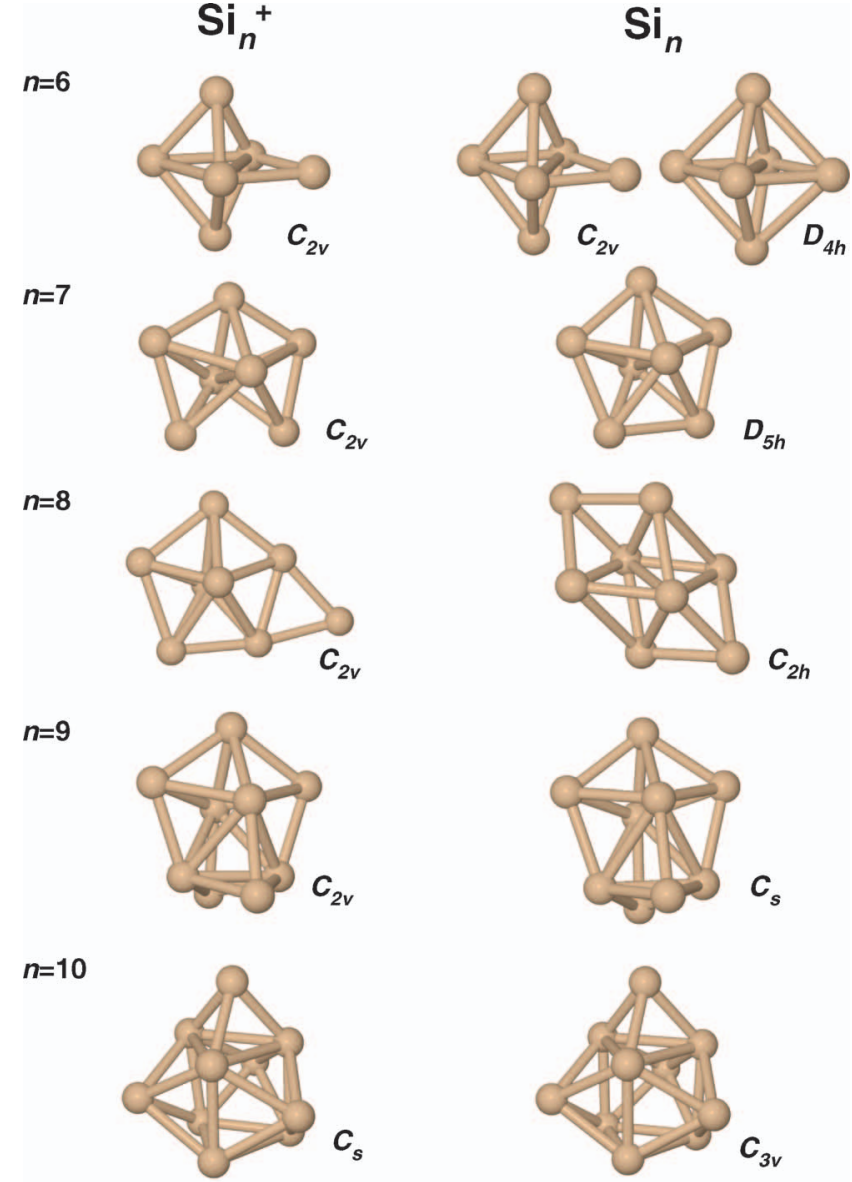

$n=15$
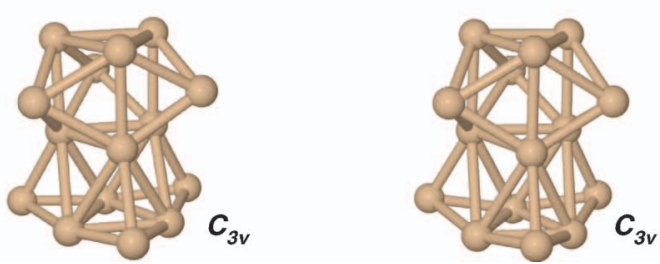

FIG. 7. Comparison between the structures determined by gas-phase IR spectroscopy for cationic and neutral silicon clusters with $n=6-10$ and $n=15$. Approximate symmetries are determined from the atomic geometries calculated without symmetry constraints.

weaker band at $\sim 485 \mathrm{~cm}^{-1}$ has no counterpart in the calculated spectrum and its origin is unclear. It may be due to the presence of the messenger atom, which is not accounted for in the calculations. None of the calculated spectra of higher energy isomers (for additional isomers see electronic supplementary information ${ }^{40}$ ) gives a better fit to the measured spectrum or can explain the additional feature. The identified ground state isomer has a large electric dipole moment of $2.9 \mathrm{D}$ that may facilitate the formation of the Xe complex. The other cluster sizes forming the messenger complex, however, have calculated dipole moments of only 0.001 D and 0.290 D, for $\mathrm{Si}_{8}$ and $\mathrm{Si}_{9}$, respectively. Apparently, the dipole moment is not the critical parameter for the complex formation.

\section{CONCLUSION}

Cluster-size specific vibrational spectra of $\mathrm{Si}_{6-10}$ and $\mathrm{Si}_{15}$ have been measured by using two different gas-phase IR 
spectroscopic methods, IR-MPD of Xe messenger complexes and IR-UV two-color ionization. Comparison with predictions from density functional theory allows assigning the clusters' structures. The identified structures correspond to the lowest energy isomers as predicted at the BP-86 level of theory. The structures of the smaller clusters $\left(\mathrm{Si}_{6-9}\right)$ are found to be based on the bipyramidal motif. $\mathrm{Si}_{6}$ has the structure of a (distorted) tetragonal bipyramid that forms, if capped by two atoms, the $\mathrm{Si}_{8}$ structure. The pentagonal bipyramid of $\mathrm{Si}_{7}$ forms the base for the $\mathrm{Si}_{9}$ cluster. With the exception of $\mathrm{Si}_{8}$, the same growth pattern as for cationic clusters is observed (see Fig. 7). Similar to cationic silicon clusters, the tricapped trigonal prism structure is not yet found for $\mathrm{Si}_{9}$, but is the fundamental motif for clusters from $\mathrm{Si}_{10}$ onwards. For $\mathrm{Si}_{8}$ a marked difference is observed between cationic and neutral structure, as they are built upon the pentagonal vs. tetragonal bipyramidal motif, respectively. This structural change can be rationalized by the bonding character of the HOMO of $\mathrm{Si}_{8}$ which relaxes the bipyramidal structure upon ionization.

Even though the structures of neutral silicon clusters have been predicted by theory before, this is the first unambiguous experimental confirmation for larger clusters. The presented results provide reference data for theoretical investigations and may allow for further insights into the charge-state dependence of the clusters' properties in general.

\section{ACKNOWLEDGMENTS}

We gratefully acknowledge the support of the Stichting voor Fundamenteel Onderzoek der Materie (FOM) for providing beam time on FELIX. The authors thank the FELIX staff for their skillful assistance, in particular Dr. B. Redlich and Dr. A.F.G. van der Meer. This work is supported by the Research Foundation - Flanders (FWO), the Flemish Concerted Action (GOA), and the Belgian Interuniversity Poles of Attraction (IAP) programs, by the Cluster of Excellence "Unifying Concepts in Catalysis" coordinated by the Technische Universität Berlin and funded by the Deutsche Forschungsgemeinschaft (DFG), as well as by the DFG within FOR 1282 (FI 893/4-1). J.T.L. thanks the Alexander von Humboldt Foundation, and P.C. thanks the Institute for the Promotion of Innovation by Science and Technology in Flanders (IWT) for financial support. We thank the reviewers for their helpful comments and for providing additional cluster structures obtained using the method reported in Ref. 20.

${ }^{1}$ G. E. Moore, Electronics 38, 114 (1965); reprint available from: Proc. IEEE 86, 82 (1998).

${ }^{2}$ M. Haselman and S. Hauck, Proc. IEEE 98, 11 (2010).

${ }^{3}$ D. Vuillaume, Proc. IEEE 98, 2111 (2010).

${ }^{4}$ R. I. Bahar, D. Hammerstrom, J. Harlow, W. H. Joyner, Jr., C. Lau, D. Marculescu, A. Orailoglu, and M. Pedram, Computer 40, 25 (2007).

${ }^{5}$ Y. Achiba, M. Kohno, M. Ohara, S. Suzuki, and H. Shiromaru, J. Electron Spectrosc. Relat. Phenom. 142, 231 (2005).

${ }^{6}$ C. S. Xu, T. R. Taylor, G. R. Burton, and D. M. Neumark, J. Chem. Phys. 108, 1395 (1998).
${ }^{7}$ J. Müller, B. Liu, A. A. Shvartsburg, S. Ogut, J. R. Chelikowsky, K. W. M. Siu, K. M. Ho, and G. Ganteför, Phys. Rev. Lett. 85, 1666 (2000).

${ }^{8}$ M. A. Hoffmann, G. Wrigge, B. von Issendorff, J. Müller, G. Ganteför, and H. Haberland, Eur. Phys. J. D 16, 9 (2001).

${ }^{9}$ G. Meloni, M. J. Ferguson, S. M. Sheehan, and D. M. Neumark, Chem. Phys. Lett 399, 389 (2004).

${ }^{10}$ S. J. Peppernick, K. D. D. Gunaratne, S. G. Sayres, and A. W. Castleman, J. Chem. Phys. 132, 044302 (2010).

${ }^{11}$ K. Fuke, K. Tsukamoto, F. Misaizu, and M. Sanekata, J. Chem. Phys. 99, 7807 (1993).

${ }^{12}$ O. Kostko, S. R. Leone, M. A. Duncan, and M. Ahmed, J. Phys. Chem. A 114, 3176 (2010).

${ }^{13}$ R. R. Hudgins, M. Imai, M. F. Jarrold, and P. Dugourd, J. Chem. Phys. 111, 7865 (1999).

${ }^{14}$ K. Raghavachari, J. Chem. Phys. 84, 5672 (1986).

${ }^{15}$ P. Ballone, W. Andreoni, R. Car, and M. Parrinello, Phys. Rev. Lett. 60, 271 (1988)

${ }^{16}$ K. Raghavachari, and C. M. Rohlfing, J. Chem. Phys. 89, 2219 (1988).

${ }^{17}$ W. von Niessen, and V. G. Zakrzewski, J. Chem. Phys. 98, 1271 (1993).

${ }^{18}$ B. X. Li, P. L. Cao, and X. Y. Zhou, Phys. Status Solidi B 238, 11 (2003).

${ }^{19}$ J. C. Yang, W. G. Xu, and W. S. Xiao, J. Mol. Struct. THEOCHEM 719, 89 (2005).

${ }^{20}$ S. Goedecker, W. Hellmann, and T. Lenosky, Phys. Rev. Lett. 95, 055501 (2005).

${ }^{21}$ S. Nigam, C. Majumder, and S. K. Kulshreshtha, J. Chem. Phys. 125, 074303 (2006).

${ }^{22}$ S. De, S. A. Ghasemi, A. Willand, L. Genovese, D. Kanhere, and S. Goedecker, J. Chem. Phys. 134, 124302 (2011)

${ }^{23}$ K. M. Ho, A. A. Shvartsburg, B. C. Pan, Z. Y. Lu, C. Z. Wang, J. G. Wacker, J. L. Fye, and M. F. Jarrold, Nature (London) 392, 582 (1998).

${ }^{24}$ B. Liu, Z. Y. Lu, B. C. Pan, C. Z. Wang, K. M. Ho, A. A. Shvartsburg, and M. F. Jarrold, J. Chem. Phys. 109, 9401 (1998).

${ }^{25}$ P. Garcia-Fernandez, J. E. Boggs, and J. F. Stanton, J. Chem. Phys. 126, 074305 (2007)

${ }^{26}$ J. T. Lyon, P. Gruene, A. Fielicke, G. Meijer, E. Janssens, P. Claes, and P. Lievens, J. Am. Chem. Soc. 131, 1115 (2009).

${ }^{27}$ W. Qin, Q.-J. Zang, W.-C. Lu, C.-Z. Wang, and K.-M. Ho, Chem. Res. Chinese U. 27, 313 (2011)

${ }^{28}$ E. C. Honea, A. Ogura, C. A. Murray, K. Raghavachari, W. O. Sprenger, M. F. Jarrold, and W. L. Brown, Nature (London) 366, 42 (1993).

${ }^{29} \mathrm{~S}$. Li, R. J. Vanzee, W. Weltner, and K. Raghavachari, Chem. Phys. Lett. 243, 275 (1995).

${ }^{30}$ A. Fielicke, J. T. Lyon, M. Haertelt, G. Meijer, P. Claes, J. de Haeck, and P. Lievens, J. Chem. Phys. 131, 171105 (2009).

${ }^{31}$ W. Bouwen, P. Thoen, F. Vanhoutte, S. Bouckaert, F. Despa, H. Weidele, R. E. Silverans, and P. Lievens, Rev. Sci. Instrum. 71, 54 (2000).

${ }^{32}$ E. Janssens, H. Tanaka, S. Neukermans, R. E. Silverans, and P. Lievens, Phys. Rev. B 69, 085402 (2004).

${ }^{33}$ M. Haertelt, A. Fielicke, G. Meijer, K. Kwapien, M. Sierka, and J. Sauer, "Structure determination of neutral $\mathrm{MgO}$ clusters-hexagonal nanotubes and cages," Phys. Chem. Chem. Phys. (in press).

${ }^{34}$ M. B. Knickelbein, and W. J. C. Menezes, Chem. Phys. Lett. 184, 433 (1991).

${ }^{35}$ A. D. Becke, Phys. Rev. A 38, 3098 (1988).

${ }^{36}$ J. P. Perdew, Phys. Rev. B 33, 8822 (1986).

${ }^{37}$ A. Schäfer, H. Horn, and R. Ahlrichs, J. Chem. Phys. 97, 2571 (1992).

${ }^{38}$ M. J. Frisch, G. W. Trucks, H. B. Schlegel et al., GAussian 03, Rev. C.02, Gaussian, Inc., Wallingford, CT, 2004.

${ }^{39}$ A. Fielicke, G. von Helden, and G. Meijer, Eur. Phys. J. D 34, 83 (2005).

${ }^{40}$ See supplementary material at http://dx.doi.org/10.1063/1.3682323 for additional isomers of $\mathrm{Si}_{9}$ and $\mathrm{Si}_{15}$, calculated IR resonances and IR intensities for the assigned ground state structures, and atomic Cartesian coordinates for $\mathrm{Si}_{8,9,15}$.

${ }^{41}$ W. Hellmann, R. G. Hennig, S. Goedecker, C. J. Umrigar, B. Delley, and T. Lenosky, Phys. Rev. B 75, 085411 (2007). 